\title{
Editorial: From innovative experiences to wider visions in higher education
}

\author{
Luigi F. Donà dalle Rose \\ Editor
}

Anna Serbati

Assistant Editor

doi: http://dx.doi.org/10.18543/tjhe-5(2)-2018pp13-16

Nowadays, there is a growing awareness that higher education is called to help young people to develop their personal and professional future. The university mission is not only to increase opportunities for employability and for better matching of labour market requests and graduates' skills, but also to prepare people to positively live in local and global communities as well as to actively contribute to personal and community well-being. Therefore, a more holistic approach to education is required, which overcomes the traditional idea of promoting logical, cognitive and linguistic intelligence and which promotes multiple intelligences, including emotional, interpersonal, creative skills. Scholarship of teaching and learning in higher education and educational research have shown that there is a variety of strategies and methods that can foster not only the development of knowledge, but also soft skills. This Issue offers some perspectives and innovative experiences in different subject areas within this framework and moves towards more general visions of educational issues.

The first three contributions offer interesting examples of innovative teaching-learning experiences face-to-face as well as online, able to promote a more holistic approach to education. The following contribution presents meditated reflections and feasable proposals for further wider engagement of the medical education operators in the field of health care. It reports findings of concerns and possible wider expectations of healthcare operators and staff. The last contribution flies high, comparing two different approaches towards harmonisation of higher education at continental level, Africa and Europe respectively.

The first article by Jorge Jaime dos Santos Fringe presents results of a study carried out with psychology students at University Eduardo Mondlane, in Mozambique. A questionnaire was administered to students with the aim 
of investigating, in a competence-based framework, to which extent different teaching methods promote multiple intelligences as superimposed to holistic learning styles. Findings show that there still is more emphasis on the development of logical and analytical skills by using more frequently methods such as lectures, debates, oriented reading, brainstorming, problem solving and seminars. However, there is a growing effort to promote holistic learning by using teaching strategies such as study visits, field work, drama and simulations, case study and experiential learning, that are linked to bodily-kinesthetic intelligence and that give students the opportunity to deal with authentic tasks and develop their competences. The author identifies the need to support teachers to understand, use and adapt teaching and learning methods to promote students' multiple intelligences.

The second article, by Tiernan Henry and John Murray, also contributes to the reflection on methods and strategies that foster the development of wide competences with specific spatial and temporal skills. Indeed, the authors present the results of an investigation of the undergraduate student perception about fieldwork, specifically in the context of the affective domain. Students of Earth and Ocean Science (EOS) at NUI Galway were asked to express their opinion before and after an activity of residential work. Findings indicate an appreciation of cognitive benefits, but also transferable, technical and social skills developed: students recognised the importance and value of sound observation and scientific rigor, enhanced their academic and social confidence as well as ability to work as a group. This study showed that field training can transform the way students think (not only academically, but also socially), by making them more independent and aware of the learning process.

The contribution by Ana Nobre describes and documents an experience of foreign language teaching and learning at Universidade Aberta (the Portuguese Open University), in Portugal. Curricular pathways proposing online oral and written communicative practices in different languages are presented. Digital resources included in online teaching comprise multimedia materials, produced by the teachers or by the students, as well as other materials available on the web 2.0. A survey administered to students showed that these online resources are perceived by students as suitable for the online teaching and learning of foreign languages, particularly for adults, and foster an improvement not only of oral discourse comprehension but also an increase of the foreign language level of knowledge.

The next article regards those aspects of the higher education staff experience, which are linked to the outer world, more concretely to existing higher education laws and directives. The contribution by Roberta Inés 
Ladenheim and Cecilia Inés Hernández deals with a current theme in several professional communities when engaged in planning teaching, learning and training activities. Their "exploratory" study stems from the absence of a "common language" regarding the conception of the terms like generic competences in the Argentinian relevant legislative and academic context of medical education (both at different institutional levels and in the academic and disciplinary milieu). The study explores which "conceptions" underlie the terms generic competences among the medical educational planners in Argentina and - again - explores the language choices they make when referring to those competences. Semi-structured interviews were conducted in Spanish between July and September 2017 among "expert" informants, carefully chosen from different levels and fields in the Argentinian environment of Health Sciences curricular planning. Results confirm the initial working hypothesis of different conceptions regarding the terms generic competences and moreover show that a given generic competence acquires a new specificity when immersed in the medical education context. The authors' conclusion is that a serious effort on consensus building about terminology is needed, at least - but perhaps not only - in medical education.

The last contribution deals with wider processes, which since two decades are animating the national higher education systems, with the aim of concretely implementing the paradigm shift which started earlier at the lower levels of education, i.e. the shift from an input-based teaching/learning methodology to student-centred approaches, noticeably competence-based learning methodologies. These processes are indeed characterised by a strong international dimension, which on the basis of fruitful comparisons among different national situations favours subsequent educational policy agreements and national reforms at sub-regional or even regional/continental level.

The contribution by Ayenachew Aseffa Woldegiyorgis discusses and compares two such convergence processes, i.e. the European process which started with the Bologna Declaration in 1999 and the African process, which is defined by the Addis Ababa convention (2014). The article gives a theoretical background for the several existing harmonisation processes, also enlightening its geopolitical aspects and the role of supranational organisations (UNESCO, World Bank, ...). Moreover, it offers detailed historical overviews of the two processes chosen for discussion. Finally, and perhaps most interestingly, it offers a parallel analysis of the European and African processes. According to one reviewer, "the author rightly notes that the African process is in full construction, compared to the European one which is today at a phase of implementation". Moreover, in his conclusions related 
to the African process, the author recognises the merits of a "sub-continental focus" because "the participating countries have more in common anchored in their geographic proximity and shared history." However, these "subregional strengths" should be capitalised by the African Union to actively "coordinate the existing efforts into a continent-wide system". 


\title{
Editorial: From innovative experiences to wider visions in higher education
}

\author{
Luigi F. Donà dalle Rose \\ Editor
}

Anna Serbati

Assistant Editor

doi: http://dx.doi.org/10.18543/tjhe-5(2)-2018pp13-16

\section{Copyright}

Copyright for this article is retained by the Publisher. It is an Open Access material that is free for full online access, download, storage, distribution, and or reuse in any medium only for noncommercial purposes and in compliance with any applicable copyright legislation, without prior permission from the Publisher or the author(s). In any case, proper acknowledgement of the original publication source must be made and any changes to the original work must be indicated clearly and in a manner that does not suggest the author's and or Publisher's endorsement whatsoever. Any other use of its content in any medium or format, now known or developed in the future, requires prior written permission of the copyright holder. 